\title{
RETRACTION
}

doi:10.1038/nature06621

\section{Anti-apoptotic function of a microRNA encoded by the HSV-1 latency-associated transcript}

A. Gupta, J. J. Gartner, P. Sethupathy, A. G. Hatzigeorgiou \& N. W. Fraser

Nature 442, 82-85 (2006)

We claimed that there was a microRNA in the exon 1 region of the latency-associated transcript of herpes simplex virus-1 (HSV-1) that downregulated the expression of transforming growth factor- $\beta$ (TGF- $\beta$ ). We postulated that through this mechanism the virus prevented the latently infected cell from apoptosing. We are confident that HSV-1 downregulates TGFB messenger RNA; however, the mapping of this downregulation to the region of the HSV-1 genome that we predicted to encode the microRNA is not supported by further experiments in several laboratories.

B. R. Cullen from Duke University and D. Coen from Harvard University have not been able to detect the microRNA that we described. Using samples that they have supplied, we also been unable to consistently detect the predicted microRNA. Furthermore, in the samples supplied by the Cullen laboratory, we could only detect miR-LAT signal in SY5Y neuronal-like cell samples and not in non-neuronal 293 cell samples. The SY5Y cells were found to be positive for miR-LAT, regardless of whether or not they were infected with HSV-1, suggesting that we are detecting a SY5Yencoded microRNA.

A.G. has declined to sign the retraction because she maintains that the original observations are correct. 